EESTI NSV TEADUSTE AKADEEMIA TOIMETISED. 25. KÖIDE

FOUSIKA * MATEMAATIKA. 1976, NR. 1

ИЗВЕСТИЯ АКАДЕМИИ НАУК ЭСТОНСКОИ ССР. ТОМ 25 ФИЗИКА * МАТЕМАТИКА. 1976, № 1

Э. $B E C M A H$

удК 530.145

\title{
К ВОПРОСУ О ЕДИНСТВЕННОСТИ СУММЫ АСИМПТОТИЧЕСКОГО РЯДА В ТЕОРИИ С НЕПОЛИНОМИАЛЬНЫМ ЛАГРАНЖИАНОМ
}

1. Будем говорить, что формальный степенной ряд

$$
\sum_{n=0}^{\infty} c_{n}(-z)^{n}
$$

является асимптотическим для функции $F(-z)$ при $z \rightarrow 0$ в секторе $\Theta_{1}$ на комплексной плоскости $z=\zeta \exp (i \Theta)$, если для любого $n \geqslant 0$

$$
\lim _{z \rightarrow 0}\left[F(-z)-\sum_{v=0}^{n} c_{v}(-z)^{v}\right] / z^{n}=0
$$

равномерно относительно всех $|\Theta|<\Theta_{1}$. Часто встречается задача нахождения $F(-z)$, т. е. суммирования ряда (1). Как известно $\left[{ }^{1}\right]$, для любой последовательности $c_{n}$ и любого $\alpha>0$ существуют функции $F(-z)$ такие, что условие $(2)$ в секторе $|\Theta| \leqslant \alpha \pi$ выполняется равномерно. Если $A>0$ и $2 k \alpha<1$, то $\zeta^{-n} \exp \left(-A z^{-k}\right) \rightarrow 0$ равномерно в указанном угле для любого $n \geqslant 0$. Тем самым все функции $F(-z)+$ $+c \exp \left(-A z^{-k}\right)$ имеют в этом секторе одно и то же асимптотическое разложение и задача суммирования последнего не имеет однозначного решения. Положение меняется, если коэффициенты $c_{n}$ выполняют определенные требования. Тогда может оказаться, что условию (2) удовлетворяет в рассматриваемом секторе не более одной аналитической функции $F(-z)$ и что она есть в некотором (например борелевском) смысле сумма данного ряда.

Можно также сформулировать необходимсе и достаточное условие для того, чтобы асимптотический ряд имел однозначно определенную сумму, опираясь на следующую теорему Карлемана $\left(\left[{ }^{2}\right]\right.$, там же см. ссылки на оригинальные работы).

Те орема. Если функция ф(z) аналитична на римановой поверхности $\ln z$ в секторе

$$
T=\left\{|z|<B:|\arg z|<\frac{m \pi}{2}\right\},
$$

где В - постоянная, а $m$ - целое число, и условие

$$
|\varphi(z)|<b_{N} \mid z^{\mid N}
$$


для любого $N \geqslant 0$ выполняется в $T$ равномерно, то для $\varphi(z) \equiv 0$ необходимо и достаточно, чтобы

$$
\sum_{N=0}^{\infty} b_{N}^{-(m N)^{-1}}=\infty
$$

Пусть теперь $F(-z)$ аналитична в $T$ и имеет асимптотическое разложение

такое, что

$$
F(-z) \sim \sum_{n=0}^{\infty} c_{n}(-z)^{n}
$$

$$
\left|R_{N}(z)\right|=\left|F(-z)-\sum_{n=1}^{N-1} c_{n}(-z)^{n}\right|<b_{N}|z|^{N}
$$

для любого $N$ и равномерно в $T$. Пусть и $L(-z)$ аналитична в $T$, имея то же самое асимптотическое разложение. Тогда для $F(-z) \equiv$ $\equiv L(-z)$ необходимо и достаточно, чтобы выполнялось условие $(5)$. Отметим, что равномерное выполнение в $T$ остаточным членом условия

$$
\left|R_{N}(z)\right|<A \varrho^{N}(m N) !|z|^{N},
$$

где $A>0$ и $\varrho>0$, обеспечит однозначность.

2. Будем рассматривать асимптотический степенной ряд

$$
\sum_{n=0}^{\infty}(m n) !(-z)^{n}
$$

Пользуясь обобщенной теоремой Ватсона $\left[{ }^{3}\right]$, можно показать, что ряд (8) суммируем методом Бореля к сумме

$$
F_{m}(-z)=\int_{0}^{\infty} \mathrm{e}^{-t}\left(1+z t^{m}\right)^{-1} \mathrm{~d} t
$$

в секторе $D=\{|z|<B:|\arg z| \leqslant \lambda\}$. Здесь $B>0$ и $0<\lambda<m \pi$. Сумма $F_{m}(-z)$ имеет аналитическое продолжение за пределы сектора $D$ и асимптотическое разложение, совпадающее с (8) во всем секторе своей регулярности.

Пусть $m=1$. Очевидно, что (9) представляет функцию, аналитическую на комплексной плоскости $z$, кроме разреза по лучу $\arg z=\pi$. Функция $F_{1}$ имеет два аналитических продолжения, которые получим, переходя на разные листы римановой поверхности $\ln z$, путем поворота контура интегрирования в (9) на $-\pi / 2$ :

и на $\pi / 2$ :

$$
F_{1}^{1}(-z)=-\mathrm{i} \int_{0}^{\infty} \mathrm{e}^{\mathrm{i} t}(1-\mathrm{i} z t)^{-1} \mathrm{~d} t
$$

$$
F_{2}^{2}(-z)=\mathrm{i} \int_{0}^{\infty} \mathrm{e}^{-\mathrm{i} t}(1+\mathrm{i} z t)^{-1} \mathrm{~d} t .
$$

Перепишем теперь (9), заменяя $z^{1 / m}=x$,

$F_{m}(-z)=Q_{m}(x)=\sum_{k=1}^{m} b_{k m} \int_{0}^{\infty} \mathrm{e}^{-t}\left(1-u_{k m} x t\right)^{-1} \mathrm{~d} t=\sum_{k=1}^{m} b_{k m} F_{1}\left(u_{k m} x\right)$,

где

$$
u_{k m}=\exp [\mathrm{i}(2 k-1) \pi / m]
$$

и $b_{k m}$ - постоянные.

Осуществим аналитическое продолжение в (12) следующим образом: те члены суммы, у которых разрез в секторе $\pi / m \leqslant \arg x<\pi / 2+\pi / m$, продолжим путем поворота контура интегрирования на $-\pi / 2$, а те 
члены, у которых разрез в секторе $-\pi / m \geqslant \arg x>-\pi / 2-\pi / m$, путем поворота контура интегрирования на $\pi / 2$, а остальные менять не будем. В результате получим сумму, которая регулярна в секторе

$$
|\arg x|<\pi / m+\pi / 2
$$

и является аналитическим продолжением суммы (12):

$Q_{m}^{A}(x)=\sum_{h=1}^{h} b_{k m} F_{1}^{2}\left(u_{k m} x\right)+\sum_{h=h+1}^{m-h} b_{k m} F_{1}\left(u_{k m} x\right)+\sum_{h=m-h+1}^{m} b_{k m} F_{1}^{1}\left(u_{k m} x\right)$

Здесь $h-$ наибольшее целое число $\leqslant 1+m / 4$. Переходя обратно к переменной $z$, заключаем, что (9) определяет регулярную функцию в секторе

Теперь покажем, что

$$
|\arg z|<\pi+m \pi / 2 \text {. }
$$

а) функция $F_{m}(-z)$ представлена в секторе $|\Theta|<\pi+m \pi / 2$, $\Theta=\arg z$ асимптотическим рядом

$$
F_{m}(-z) \sim \sum_{n=0}^{N-1}(m n) !(-z)^{n}+R_{N}(z) ;
$$

б) $\left|R_{N}(z)\right|<A Q^{N}(m N) !|z|^{N}$ равномерно для $N$ и $|\Theta|<\pi+m \pi / 2-\delta$, $\delta>0$.

Пусть опять $m=1$. Тогда

$$
F_{1}(-z) \sim \sum_{n=0}^{N-1} n !(-z)^{n}+R_{N}(z), \quad|\Theta|<\pi,
$$

где

$$
\begin{gathered}
\left|R_{N}(z)\right|<N !|z|^{N}, \quad|\Theta|<\pi / 2, \\
\left|R_{N}(z)\right|<N !|z|^{N}|\sin \Theta|^{-1}, \quad \pi / 2<|\Theta|<\pi .
\end{gathered}
$$

Пределы (18)-(19) можно получить, воспользовавшись в (9) тождеством

$$
(1+t z)^{-1}=\sum_{n=0}^{N-1}(-t z)^{n}+(-t z)^{N}(1+t z)^{-1},
$$

где

$$
\begin{array}{ll}
\left|(1+t z)^{-1}\right|<1, & |\Theta|<\pi / 2, \\
\left|(1+t z)^{-1}\right|<\left|(\sin \Theta)^{-1}\right|, & \pi / 2<|\Theta|<\pi .
\end{array}
$$

Пределы действительны равномерно в любом секторе $|\Theta|<\pi-\varepsilon$, $\varepsilon>0$. Теперь будем поворачивать контур интегрирования в $(9)(m=1)$ соответственно на $-\pi / 2+\varepsilon$ и на $\pi / 2-\varepsilon$. Так как полученные интегралы можно рассматривать как первоначальные, то асимптотическое разложение (17) действительно и в секторе $|\Theta|<3 \pi / 2$ и имеет ограниченный остаточный член

$$
\left|R_{N}(z)\right|<N !|z|^{N}|\sin \varepsilon|^{-N-1}\left|\sin \varepsilon_{1}\right|^{-1}<A Q^{N} N !|z|^{N}
$$

равномерно в секторе $|\Theta|<3 \pi / 2-\left(\varepsilon+\varepsilon_{1}\right), \varepsilon>0, \varepsilon_{1}>0$, для некоторых положительных $A$ и $\varrho$.

Общий случай $m>1$ может быть рассмотрен аналогично, поскольку $F_{m}$ является линейной комбинацией от $F_{1}$. И, таким образом, придем к заключениям а) и б).

Разницу между функцией $F_{1}$ и ее аналитическими продолжениями вычислим с помощью теоремы о вычетах:

$$
F_{1}(-z)-F_{1}^{1}(-z)= \begin{cases}0, & -\pi / 2<\arg z<\pi \\ -2 \pi i \exp (1 / z), & \pi \leqslant \arg z<3 \pi / 2\end{cases}
$$




$$
F_{1}(-z)-F_{1}^{2}(-z)= \begin{cases}0, & \pi / 2>\arg z>-\pi \\ 2 \pi \mathrm{i} \exp (1 / z), & -\pi \geqslant \arg z>-3 \pi / 2\end{cases}
$$

В общем случае $m>1$ соответствующая формула слишком громоздка. Поэтому запишем ее для примера только в случае $m=5$ (учитывая, что $F_{m}(-z)=Q_{m}(x), x=z^{1 / 5}$, а $Q_{5}$ регулярна в секторе $|\arg x|<$ $<7 \pi / 10)$ :

$$
Q_{5}(x)-Q_{5}^{A}(x)=\left\{\begin{array}{cc}
0, & |\arg x|<\pi / 5, \\
2 \pi \mathrm{i} b_{15} E_{15}, & -\pi / 5 \geqslant \arg x>-3 \pi / 5, \\
-2 \pi \mathrm{i} b_{15}^{*} E_{15}^{*}, & \pi / 5 \leqslant \arg x<3 \pi / 5, \\
2 \pi \mathrm{i}\left[b_{15} E_{15}+b_{25} E_{25}\right], & -3 \pi / 5 \geqslant \arg x>-7 \pi / 10, \\
-2 \pi \mathrm{i}\left[b_{15}^{*} E_{15}^{*}+b_{25}^{*} E_{25}^{*}\right], & 3 \pi / 5 \leqslant \arg x<7 \pi / 10 .
\end{array}\right.
$$

Здесь введено обозначение $E_{k m}=\exp \left(-1 / x u_{k m}\right), u_{k m}$ дается (13) и звездочка означает комплексное сопряжение.

3. В теории поля с неполиномиальными лагранжианами $\left[{ }^{4}\right]$ встречается задача суммирования асимптотического ряда

$$
\sum_{n=0}^{\infty}(m n) ! \xi^{n}, \quad \xi=|z| .
$$

Из предыдущего ясно, что сумма ряда $(25) F_{m}(\xi)$ может быть получена путем аналитического продолжения суммы (9) соответствующего знакопеременного ряда (8), которая определена на комплексной плоскости $z$ везде однозначно, кроме разреза вдоль положительной действительной оси. Как показывают формулы (22)-(24), продолжение функции (9) на положительные действительные $z$ оказывается многозначной комплексной функцией, которая имеет на разных ветвях попарно комплексно сопряженные значения и приобретает при переходе через разрез на римановую поверхность $\ln z$ добавку в виде функции, у которой существенная особенность в нуле. Чем быстрее коэффициенты ряда (25) растут, тем больше сектор регулярности ее борелевой суммы на римановой поверхности $\ln z$ (см. (16)) и тем больше количество ветвей. При $m=1$ аналитическое продолжение (9) на положительные действительные $z$ имеет две ветви, при $m \geqslant 5$ появляются еще две, при $m \geqslant 9$ новые две и т. д.

Комплексность суммы асимптотического ряда (25) $F_{m}(\xi)$ препятствует обеспечению унитарности теории $\left[{ }^{2}\right]$, а так как для любой физической теории унитарность обязательна, то остается только принять, что сумма ряда $(25) F_{m}(\xi)$ не является физической функцией и определить физическую функцию таким образом, чтобы она была действительной при действительных положительных значениях аргумента. Этому требованию удовлетворяет линейная комбинация

$$
\begin{aligned}
& \bar{F}_{m}(\xi)=(a-\mathrm{i} b) F_{m}(\xi+\mathrm{i} 0)+(a+\mathrm{i} b) F_{m}(\xi-\mathrm{i} 0)= \\
= & 2 a \mathrm{P} \int_{0}^{\infty} \mathrm{e}^{-t}\left(1-t^{m} \xi\right)^{-1} \mathrm{~d} t+2 \pi b / m \xi^{-1 / m} \exp \left(-\xi^{-1 / m}\right),
\end{aligned}
$$

где $\mathrm{P}$ - знак главного значения интеграла по Коши, $a$ и $b-$ произвольные действительные постоянные. Таким образом, наложение из физических соображений требования действительности функции $\bar{F}_{m}(\xi)$ внесет в теорию произвол. Функция $\bar{F}_{m}(\xi)$ не является суммой асимптотического ряда (25) в смысле, определенном в разделе 2, однако можно требовать совпадения асимптотического разложения ее с рядом (25), 
фиксируя тем самым $a=1 / 2\left[{ }^{5}\right]$. Для фиксирования постоянной $b$ необходимо привлекать дополнительные физические соображения, так как она остается свободной.

\section{ЛИТЕРАТУ РА}

1. Х а рди Г., Расходящиеся ряды, M., 1958.

2. Graffi S., Grecchi V., Phys. Rev., 6D, 493 (1972)

3. Graffi S., Grecchi V., Simon B., Phys. Lett., 32B, 631 (1970).

4. Ef i m ov G. V., Preprint ČERN, TH. 1087 (1969); E f i m o v G. V., in Proceedings of the 15th Int. Conf. on High Energy Physics, Kiev, 1970, p. 609.

5. Lee B. W., Z u m i no B., Nucl. Phys,, 13B, 671 (1969).
Ннститут физики
Академии наук Эстонской ССР
Поступила в редакцию
6/II 1975

\section{E. VESMAN}

\section{ASUMPTOOTILISE REA SUMMA UHESUSEST MITTEPOLUNOOMSE LAGRANZIAANIGA TEOORIAS}

Mittepolünoomsete interaktsioonilagranžiaanidega väljateoorias esineb asümptootiliste ridade summeerimise ülesanne. Summa on tavaliselt määratud sellise meelevaldse aditiivse funktsiooni täpsusega, millel on oluline iseärasus nullpunktis. Kuid mõnda tüüpi lagranžiaanide genereeritud asümptootilistel ridadel (25) on üheselt määratud analüütiline summa argumendi komplekstasandi mittefüüsikalises piirkonnas. Lähtudes asümptootilise rea (8) summast (9), jätkame viimast analüütiliselt füüsikalisse piirkonda (selleks on reaaltelje positiivne pool). Jätkamine on võimalik $\ln (-z)$ Riemanni pinnal kaht erinevat teed mööda. Seega osutub summa füüsikalises piirkonnas mitmeseks kompleksseks funktsiooniks, samal ajal kui teooria unitaarsuse tingimuste täitmiseks on vaja, et ta oleks reaalne. Unitaarsuse päästmiseks defineeritakse füüsikaline funktsioon sum. ma kahe haru reaalse lineaarkombinatsioonina (26). Kaasnevat meelevaldsust saab vähendada, kui nõuda füüsikalise funktsiooni (26) asümptootilise arenduse kokkulangemist reaga (25).

\section{E. VESMAN}

\section{ON THE UNIQUENESS QUESTION OF THE SUM OF THE ASYMPTOTIC EXPANSION IN THE THEORY WITH NONPOLYNOMIAL LAGRANGIAN}

Working at nonpolynomial interaction Lagrangians, one encounters the problem of the summation of asymptotic expansions. The sum is usually determined with great ambiguity - up to an additive function with an essential singularity at the origin. But some types of Lagrangians give rise to expansions (25) which have uniquely determined analytic sums in the unphysical region. In this case we start with an asymptotic expansion (8) in the unphysical region of the complex plane (where it is summable) and continue the sum (9) analytically to the physical region (which happens to be the positive real axis). The continuation is possible on the Riemann surface of $\ln (-z)$ in two different ways, and the sum in the physical region is a multivalued complex function. However, the unitarity requirements demand it to be real. Then the physical function is defined (with great ambiguity) as a real linear combination (26) of two branches of the sum. The ambiguity can be reduced by the requirement that the asymptotic expansion of the physical function (26) must coincide with the expansion (25). 